\title{
Response of free standing flexible cylinders to waves
}

\author{
D. D. K. Anbiah ${ }^{1} \&$ J. M. Roesset ${ }^{2}$ \\ ${ }^{1}$ University of Texas, Austin, Texas, USA \\ ${ }^{2}$ Texas A\&M University, College Station, Texas, USA
}

\begin{abstract}
The effects of the surrounding water on the dynamic response of flexible, free standing, cylinders fixed at their base, is studied using linear diffraction theory and two different solution approaches. The first one uses a consistent transmitting boundary to reproduce the water, while the second expresses analytically the solution in terms of the natural modes of vibration of the water and of the structure. This approach, used first by Liaw and Chopra (Report EERC 73-25, University of California, 1973) proves to be very efficient and accurate. Results are presented in terms of change in the effective natural period of the cylinder due to the presence of the water, and the effective damping, considering both an incompressible and a compressible fluid. The same formulation is used next to determine the dynamic response of these cylinders to wave action. The results (base shear and overturning moment) for the flexible cylinders are compared to those that would be obtained assuming a rigid cylinder (only diffraction forces).
\end{abstract}

Keywords: hydrodynamics, diffraction forces, flexible cylinders.

\section{Introduction}

The dynamic response of a flexible vertical cylinder fixed at its base and surrounded by water has been a problem of interest in relation to the design of some harbor structures subjected to wave action, impact forces or earthquake excitation. It was investigated in this work considering the cylinder subjected to a harmonic force applied at its top and under the action of waves. Solutions were obtained for both an incompressible and a compressible fluid, using linear diffraction theory and the equivalent of Morison's equation and comparing the numerical predictions. In the former case two different formulations were used 
and implemented in computer programs. The first one was similar to that proposed by Liaw and Chopra [1] for the seismic analysis of cylindrical towers in water. It is based on an expansion of the solution for the structural response in terms of its modes of vibration in air, and an expansion of the hydrodynamic forces in terms of the modes of the water. It has the advantage that it allows to take a different number of modes for each of the two components (structure and water).The second formulation was based on a finite element discretization of the cylinder and a semi-discrete, finite element type model of the water in terms of a consistent boundary matrix as used by Syriopoulou [2] and Hsu [3]. This formulation is analogous to that used by Blaney et al [4] for the dynamic analysis of a pile embedded in soil using the consistent soil boundary matrix developed by Kausel and Roesset [5] in cylindrical coordinates. The results of both formulations were essentially identical using ten modes for the water and 3 for the structure with the first one, and ten elements for the second, but the first approach was found to be much more economical. As a result it was the one used for an extensive series of parametric studies. The details of the formulation can be found in Anbiah [6]. In this paper we shall concentrate, due to space limitations, on the discussion of the results of the study.

\section{Vibrating cylinder under harmonic force at top}

A simple approximation to study this case is the use of Morison's equation with the water particle velocities and accelerations equal to those of the structure and a linearized drag term. The inertia and drag coefficients $C_{m}$ and $C_{d}$ are obtained from the consideration of a circular disk vibrating in a compressible or incompressible medium (plane problem). For a steady state harmonic excitation and response the equation of motion in the frequency domain becomes then

$$
E I^{\text {iv }}-\omega^{2}\left(m+C_{m} \rho \pi R^{2}\right) y+C_{d} \rho R \delta y / \delta t=P \delta(L)
$$

where $\mathrm{E}$ is the modulus of elasticity of the cylinder, I its cross sectional moment of inertia, $\mathrm{R}$ its radius, $\mathrm{L}$ its length, and $\mathrm{m}$ its mass per unit length. $\mathrm{P}$ is the amplitude of the applied force, $\omega$ the frequency of vibration, $\mathrm{y}$ the transverse displacement, $\delta y / \delta$ t the velocity, and the coefficients $\mathrm{C}_{m} \mathrm{C}_{\mathrm{d}}$ are given by

$$
\mathrm{C}_{\mathrm{m}}=-\mathrm{S}_{1}\left(\mathrm{a}_{0}\right) \quad \mathrm{C}_{\mathrm{d}}=\pi \omega \mathrm{R} \mathrm{S}_{2}\left(\mathrm{a}_{0}\right)
$$

with

$$
\mathrm{a}_{0}=\omega \mathrm{R} / \mathrm{c}_{\mathrm{w}}
$$

$\mathrm{a}_{0}$ is a dimensionless frequency, $\mathrm{c}_{\mathrm{w}}$ is the wave propagation velocity in water, of the order of $1500 \mathrm{~m} / \mathrm{s}$, and $\mathrm{S}_{1}\left(\mathrm{a}_{0}\right) \mathrm{S}_{2}\left(\mathrm{a}_{0}\right)$ are the real and imaginary parts of $\mathrm{H}_{1}$ $\left(a_{0}\right) /\left(a_{0} H^{\prime}\left(a_{0}\right)\right) \cdot H_{1}\left(a_{0}\right)$ is the Hankel function of the first order and second kind (that decays as $\mathrm{a}_{0}$ tends to infinity) and $\mathrm{H}^{\prime}\left(\mathrm{a}_{0}\right)$ is its first derivative.

Under these assumptions calling $\omega_{\mathrm{i}}$ the ith natural frequency of the cylinder in air, $\Omega_{\mathrm{i}}$ the corresponding frequency in water and $D_{\mathrm{ei}}$ the effective damping at the ith frequency one would have for compressible water

$$
\begin{gathered}
\left(\Omega_{\mathrm{i}} / \omega_{\mathrm{i}}\right)^{2}=1 /\left(1-\mathrm{RR}^{*} \mathrm{~S}_{1}\left(\mathrm{a}_{0}\right)\right) \\
\mathrm{D}_{\mathrm{ei}}=0.5\left(\Omega_{\mathrm{i}} / \omega_{\mathrm{i}}\right)^{2} \mathrm{RR}^{*} \mathrm{~S}_{2}\left(\mathrm{a}_{0}\right)
\end{gathered}
$$


where RR is the ratio of the effective density of the cylinder (its mass per unit length divided by the total cross sectional area $\pi R^{2}$ ) and the density of the water.
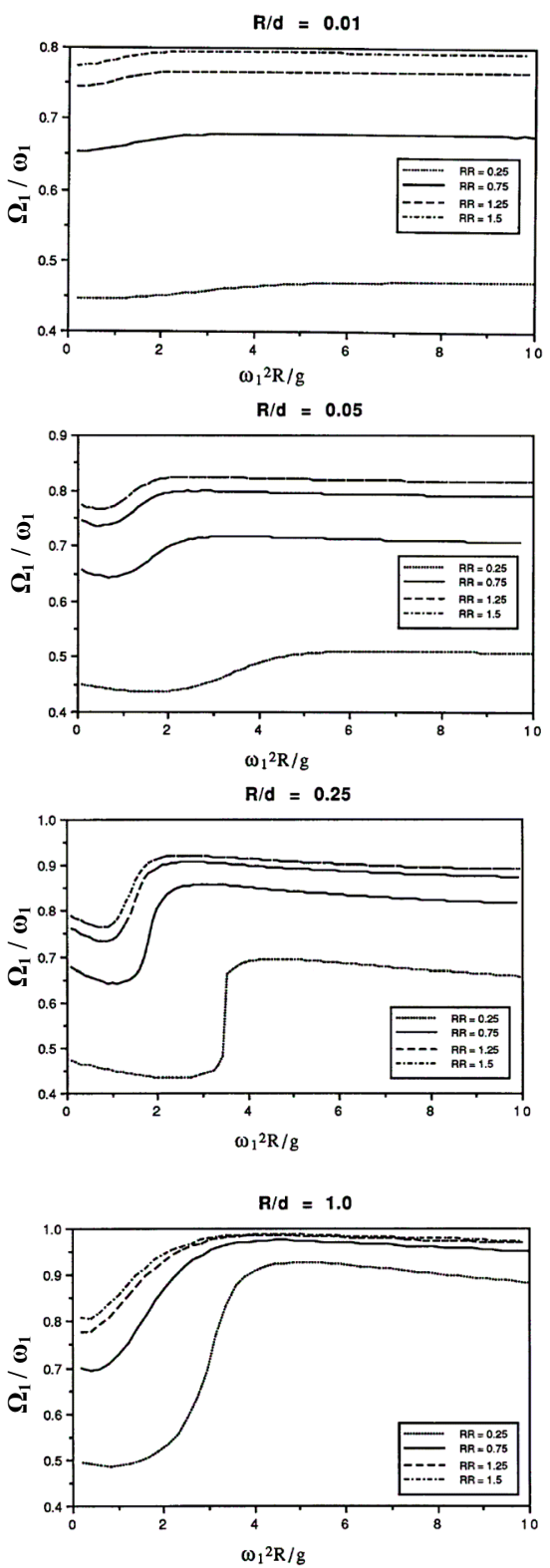

Figure 1: Variation of the effective natural period with $\omega_{1}^{2} \mathrm{R} / \mathrm{g}$ for different values of $\mathrm{R} / \mathrm{d}$. 
Considering instead the exact diffraction theory solution for the flexible cylinder in water the change in the natural frequency and the effective damping will be functions not only of the dimensionless frequency $\mathrm{a}_{0}$ and of the density ratio $R R$, but also of the slenderness ratio $d / R$ (where $d$ is the water depth) and of the factor $\omega^{2} \mathrm{R} / \mathrm{g}$ where $\mathrm{g}$ is the acceleration of gravity.
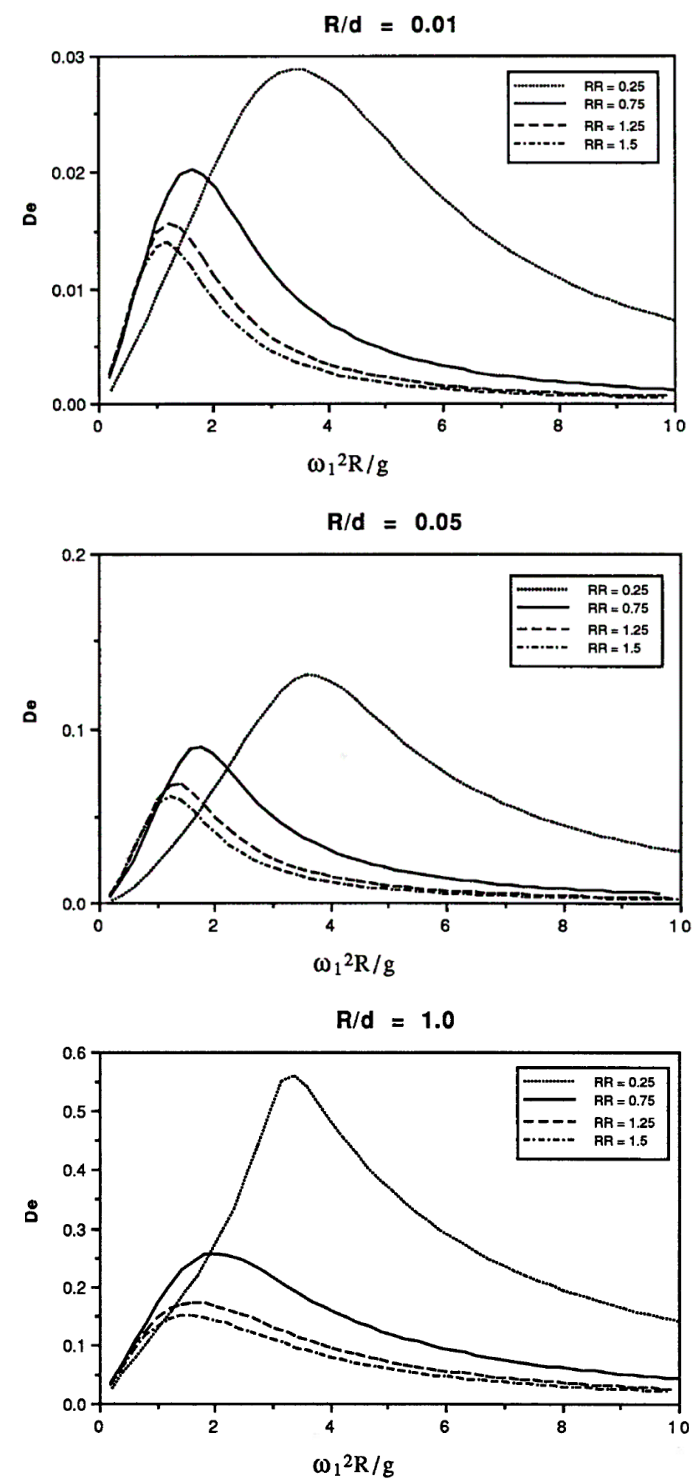

Figure 2: Variation of the effective damping with $\omega_{1}^{2} \mathrm{R} / \mathrm{g}$ for different values of R/d - Incompressible fluid. 
Figure 1 shows the variation of the ratio $\Omega_{1} / \omega_{1}$ as a function of $\omega_{1}{ }^{2} \mathrm{R} / \mathrm{g}$ for different values of the density ratio and slenderness ratios of $0.01,0.05,0.25$ and considering incompressible water. The corresponding results for a compressible fluid with values of $\omega_{1} \mathrm{R} / \mathrm{c}_{\mathrm{w}}$ ranging from 0.1 to 1 are nearly identical. It can be observed that as the value of $\mathrm{R} / \mathrm{d}$ increases, with decreasing slenderness ratio, the change in the natural frequency becomes more dependent on the value of $\omega_{1}{ }^{2} \mathrm{R} / \mathrm{g}$ particularly for low values of this factor.
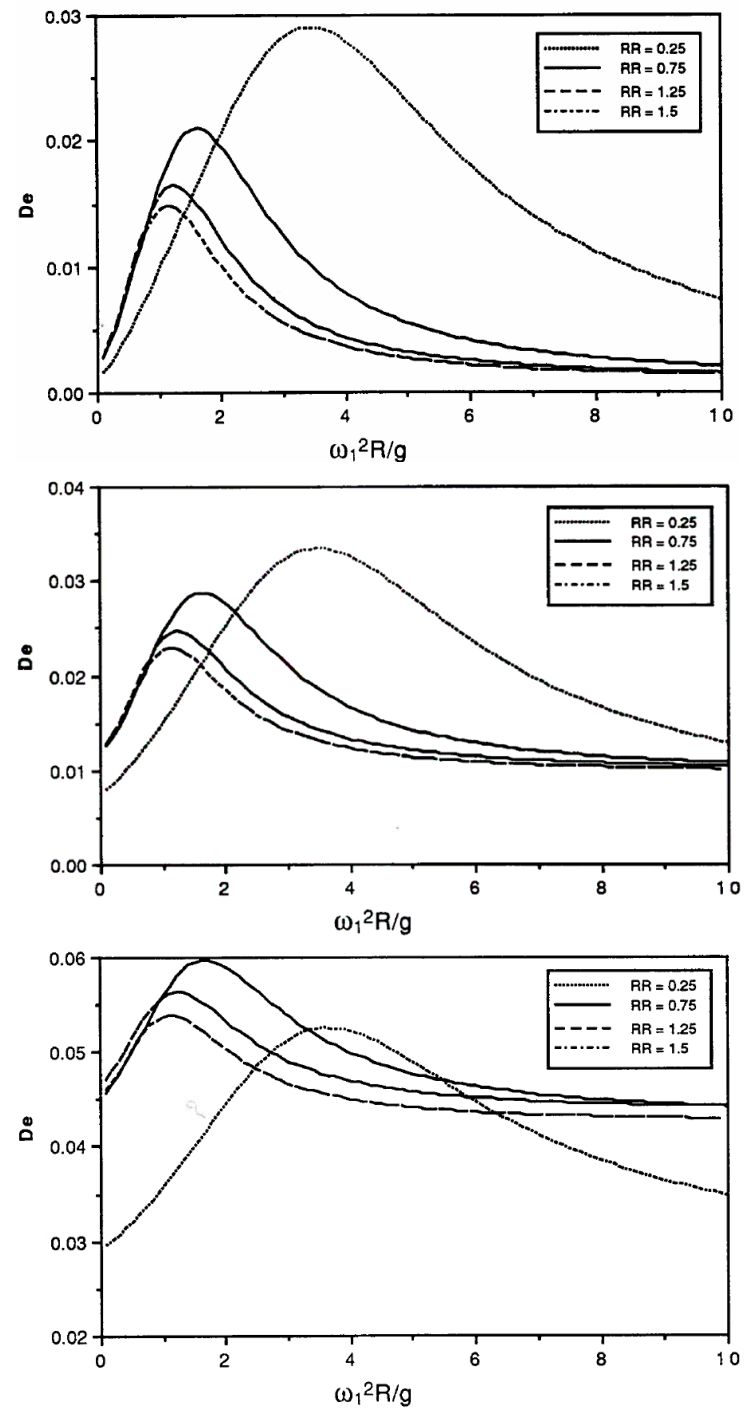

Figure 3: Variation of the effective damping with $\omega 12 \mathrm{R} / \mathrm{g}$ for $\mathrm{R} / \mathrm{d}=0.01$ and $\omega 1 \mathrm{R} / \mathrm{cw}=0.1,0.25$ and 0.5 . 
Figure 2 shows the effective damping at the first natural frequency for an incompressible fluid as a function of $\omega_{1}{ }^{2} \mathrm{R} / \mathrm{g}$ for different values of $\mathrm{R} / \mathrm{d}$. The effective damping increases in all cases as the density ratio $\mathrm{RR}$ decreases. It increases initially with increasing $\omega_{1}{ }^{2} \mathrm{R} / \mathrm{g}$ reaching a peak at a value of this factor varying approximately from 1 to 4 depending on the density ratio. It decreases then steadily for larger values of the factor. The peak values for $\mathrm{RR}=0.25$ are of the order of $3 \%$ for $\mathrm{R} / \mathrm{d}=0.01,13 \%$ for $\mathrm{R} / \mathrm{d}=0.05,45 \%$ for $\mathrm{R} / \mathrm{d}=0.25$, and $55 \%$ for $\mathrm{R} / \mathrm{d}=1$. There are therefore ranges of values of $\omega_{1}{ }^{2} \mathrm{R} / \mathrm{g}$ for which the effective damping could become significant, particularly for small slenderness ratios. For values of $\mathrm{R} / \mathrm{d}$ of 0.25 or larger the effect of the compressibility of the water on the effective damping at the fundamental frequency is again negligible. On the other hand for small values of $\mathrm{R} / \mathrm{d}$ there is a significant change in the variation of the damping with $\omega_{1}{ }^{2} \mathrm{R} / \mathrm{g}$ as the factor $\omega_{1}$ $\mathrm{R} / \mathrm{c}_{\mathrm{w}}$ increases. The change is particularly significant for the higher values of the density ratio (of the order of 1 ) as shown in Figure 3 for $\mathrm{R} / \mathrm{d}=0.01$. The results for $\mathrm{R} / \mathrm{d}=0.05$ exhibit a similar trend but the changes are less dramatic.

\section{Flexible cylinder under wave action}

To assess the effect of the flexibility of the structure on the hydrodynamic forces and the dynamic response to wave action four hypothetical cylinders were selected ranging from a very flexible one in deep water to a very stiff one in shallow water. The results for all cases were the base shear divided by $\rho G R^{2} h$ where $h$ is the wave height, the overturning moment at the base divided by $\rho \mathrm{G}$ $\mathrm{R}^{2} \mathrm{~h} \mathrm{~d}$, where $\mathrm{d}$ is the water depth and the top lateral displacement divided by the wave height. All three quantities are plotted versus $\omega^{2} \mathrm{R} / \mathrm{g}$. For the base shear and the overturning moment the results for the flexible cylinder are plotted versus the corresponding results for a rigid cylinder. Because of the normalization the relation between wave height and frequency is ignored in these figures. To get a more clear definition of the actual forces as a function of frequency one would have to select a functional relationship between the wave height and its frequency. For the rigid cylinder the displacement would be zero.

Figure 4 shows the results for the very flexible cylinder. The ratio $\mathrm{R} / \mathrm{d}$ is 0.01 , the natural frequency in air is $1 \mathrm{rad} / \mathrm{s}, \mathrm{RR}=1.5, \omega_{1} \mathrm{R} / \mathrm{c}_{\mathrm{w}}=0.0004$ and $\omega_{1}^{2} \mathrm{R} / \mathrm{g}=0.06$. All three quantities (base shear, overturning moment and displacement) show clearly two peaks associated with the first two natural frequencies of the cylinder in water. The forces at these frequencies are greatly amplified while there is an intermediate range of frequencies over which deamplification occurs with forces smaller than those that would take place for a rigid cylinder.

Figure 5 shows the corresponding results for the second cylinder with $\mathrm{R} / \mathrm{d}=0.05$ and values of the other parameters of $2 \mathrm{rad} / \mathrm{s}, 1.5,0.01$ and 3 respectively. There are acain two peaks but now the first one is associated with the variation of the hydrodynamic forces on a rigid cylinder, while the second corresponds to the first natural frequency of the cylinder in water. The results for the third cylinder with $\mathrm{R} / \mathrm{d}=0.1$, a natural frequency in air of $4.4 \mathrm{rad} / \mathrm{s}$, a density 
ratio of 0.75 and values of the other 2 parameters equal to 0.05 and 30 respectively were very similar with the second peak, corresponding to the natural frequency of the cylinder in water appearing at a higher frequency and with a smaller amplitude, as could be expected. The fourth cylinder had a value of $\mathrm{R} / \mathrm{d}=0.25$, a natural frequency in air of $22 \mathrm{rad} / \mathrm{s}$, and a density ratio of 0.75 . The forces for this case were essentially the same as for a rigid cylinder. The shape of the displacement curve was essentially the same as that of the forces with a peak amplitude of under $\mathrm{h} / 1000$.
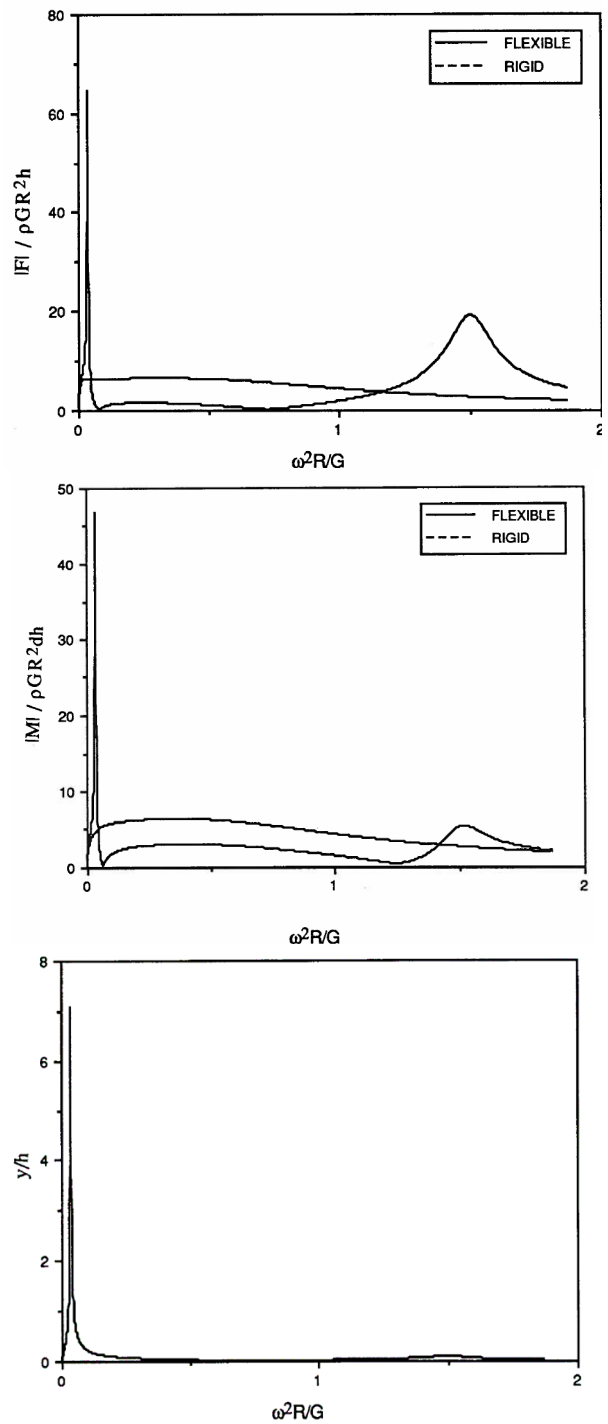

Figure 4: Base shear, overturning moment and top displacement for flexible cylinder (cylinder 1). 
82 Fluid Structure Interaction and Moving Boundary Problems IV
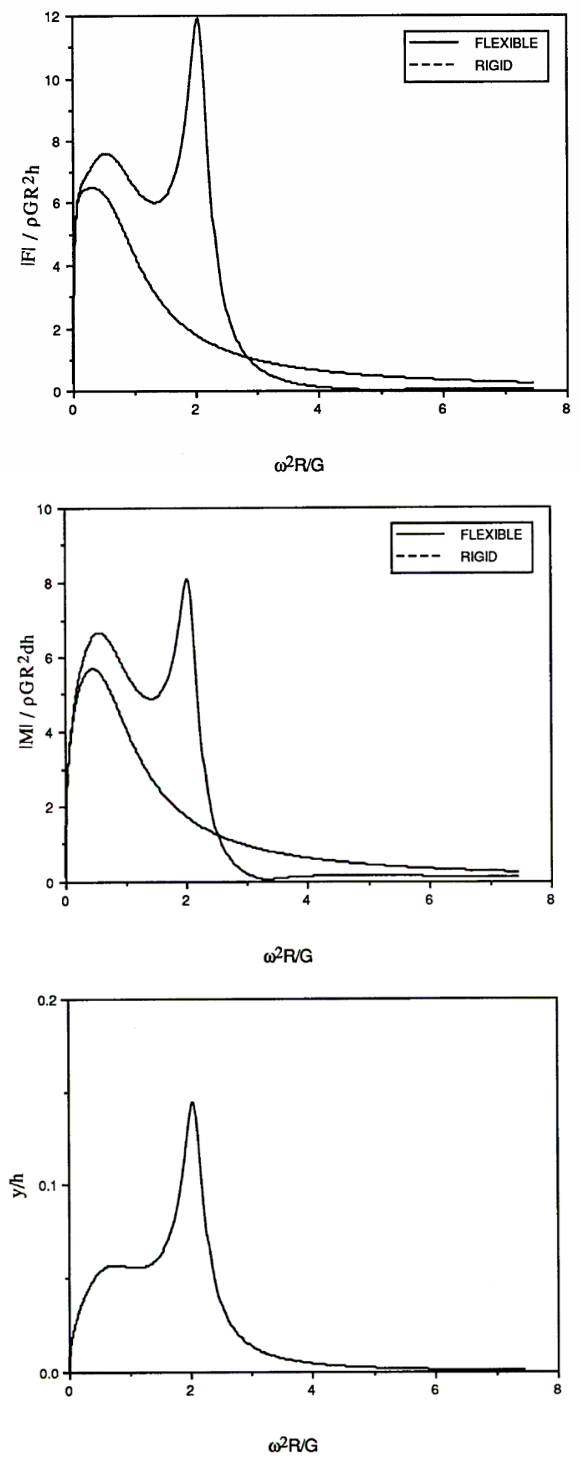

Figure 5: Base shear, overturning moment and top displacement for intermediate cylinder (cylinder 2).

\section{Conclusions}

The results of the study are as follows.

- The use of a Morison's equation type solution produced very reasonable results for the very flexible cylinders with small values of $\mathrm{R} / \mathrm{d}$ (large 
slenderness ratios) but could introduce significant errors for stiffer cylinders with larger $\mathrm{R} / \mathrm{d}$ ratios.

- The effect of the compressibility of the water was generally negligible except for the effective damping of cylinders with small values of $\mathrm{R} / \mathrm{d}$ and density ratios of the order of unity.

- The flexibility of the cylinder can affect significantly its dynamic response to waves.

\section{References}

[1] Liaw, C. Y. and Chopra, A. K. (1973) "Earthquake Response of Axisymmetric Tower Structures Surrounded by Water" Report EERC 73-25, University of California at Berkeley, Berkeley, California.

[2] Syriopoulou D. (1983) "A Numerical Technique for the Analysis of Water Wave Diffraction" M. S. Thesis, The University of Texas at Austin, Austin, Texas

[3] Hsu, Yea Chane (1984) "Diffraction Analysis for Large Axisymmetric Offshore Structures" PhD. Dissertation, The University of Texas at Austin, Austin, Texas

[4] Blaney, G.W., Kausel, E, and Roesset, J. M. (1976). "Dynamic Stiffness of Piless", ASCE, Blacksburg, Virginia.

[5] Kausel E. and Roesset, J.M. (1975) "Dynamic Stiffness of Circular Foundations", with, Journal of the Engineering Mechanics Division, ASCE

[6] Anbiah, D. D. K. (1990), "Hydrodynamic Forces on Vertical Cylinders." M.S. Thesis, The University of Texas at Austin, Austin, Texas. 